\title{
Challenges in Phase Plate Product Development
}

\author{
Bart Buijsse ${ }^{1}$, Gijs van Duinen ${ }^{1}$, Kasim Sader ${ }^{1}$, and Radostin Danev ${ }^{2}$ \\ 1. FEI, Achtseweg Noord 5, 5651 GG Eindhoven, The Netherlands \\ 2. Max Planck Institute of Biochemistry, Am Klopferspitz 18, 82152 Martinsried, Germany
}

The use of a phase plate in electron microscopy has shown renewed interest, triggered by a publication on this topic in 2001 by Danev and Nagayama [1]. This interest can be understood from the fact that many samples that are studied in TEM are weak phase objects. The use of a phase plate is the obvious method of choice to convert otherwise invisible phase modulations into visible amplitude modulations in the detected intensity profile. A phase plate can provide in-focus phase contrast, unlike the conventional method where a strong defocus is needed to generate contrast at low spatial resolutions, with the added consequence of introducing contrast reversals as a function of frequency.

Although the basic principle of a phase plate is well understood and phase contrast light microscopy is widespread it has turned out to be technologically challenging to perfect this technique in electron microscopy. The main hurdles in making a device that performs well is the sensitivity of the electron beam for any disturbances in or near the back focal plane of the microscope, the plane where the phase plate is inserted, and the strong interaction of electrons with phase plate materials Disturbances are caused by charging, contamination, and deterioration or aging of the phase plate materials due to the electrons that impinge on the device. The result is a distorted wave front of the electron beam, instead of a well-controlled phase shift between the unscattered and scattered electrons.

Many types of phase plates have been proposed and the most successful one is still the original thin-film Zernike phase plate. This type of phase plate has shown convincing performance, especially in life science applications. The most widely tested film is amorphous carbon, but this type of film suffers from aging problems, making frequent exchanges of the phase plate necessary. Alternatives to amorphous carbon have been investigated and silicon-based films show promise in terms of longevity [2].

At FEI we have investigated new methods of coming to a workable phase plate solution, addressing the problems that are still associated with phase plate usage. The work was done in collaboration with the Max Planck Institute of Biochemistry, with emphasis on practical aspects of phase plate usage in the prevailing workflow in cryo electron microscopy.

In the course of this collaboration program we have developed a new type of phase plate with properties that make it very suitable for implementing it as a user friendly device in our TEMs. Foremost, it produces high-contrast images, providing excellent contrast transfer in the low resolution range which is particularly relevant for cryo-electron tomography. No fringing effects around high-contrast features are observed and CTF oscillations can be avoided up to high spatial resolution. Also, transmission losses by the phase plate are very modest. Moreover, the phase plate shows uncompromised performance for at least half a year of usage. Finally, the phase plate allows for automated use in real-life applications such as tomography and single particle acquisition. 
The new phase plate has been characterized in detail and the presentation will deal with the properties that are specific for this type of phase plate. The phase plate holder contains a heater element to avoid contamination of the phase plate. Challenges were encountered in the construction of a heater element that fits in the restricted space between microscope pole piece and the cryo box that protects the specimen. The heater element provides excellent thermal stability to avoid phase plate drift and associated artifacts in the image during long-term data collection.

To facilitate routine phase plate usage we have added extra alignments and control panels to the microscope software. Step-by-step guided alignments have been added to set up proper illumination conditions for the phase plate. Also, highly accurate adjustment of beam deflection pivot points is taken care of to ensure a stable beam position at the plane of the phase plate. Furthermore, software was developed to easily navigate the phase plate in the back focal plane. All together, these alignments ensure seamless integration of the phase plate in the (automated) applications.

The FEI phase plate has been tested in various applications. Especially in low-dose cryo-electron tomography our phase plate has proven its added value, but also in Single Particle analysis good results have been obtained. In this talk a selection of results will be shown from cryo electron tomography work.

The use of a phase plate, especially when combined with a highly sensitive direct electron detector, will give a tremendous increase in the image contrast of weak phase objects such as unstained cryo specimens.

[1] R. Danev, K. Nagayama, Ultramicroscopy 88, 243-252 (2001)

[2] M. Marko, X. Meng, C. Hsieh, J. Roussie, C. Striemer, J. Struct. Biol. 184, 237-244 (2013)
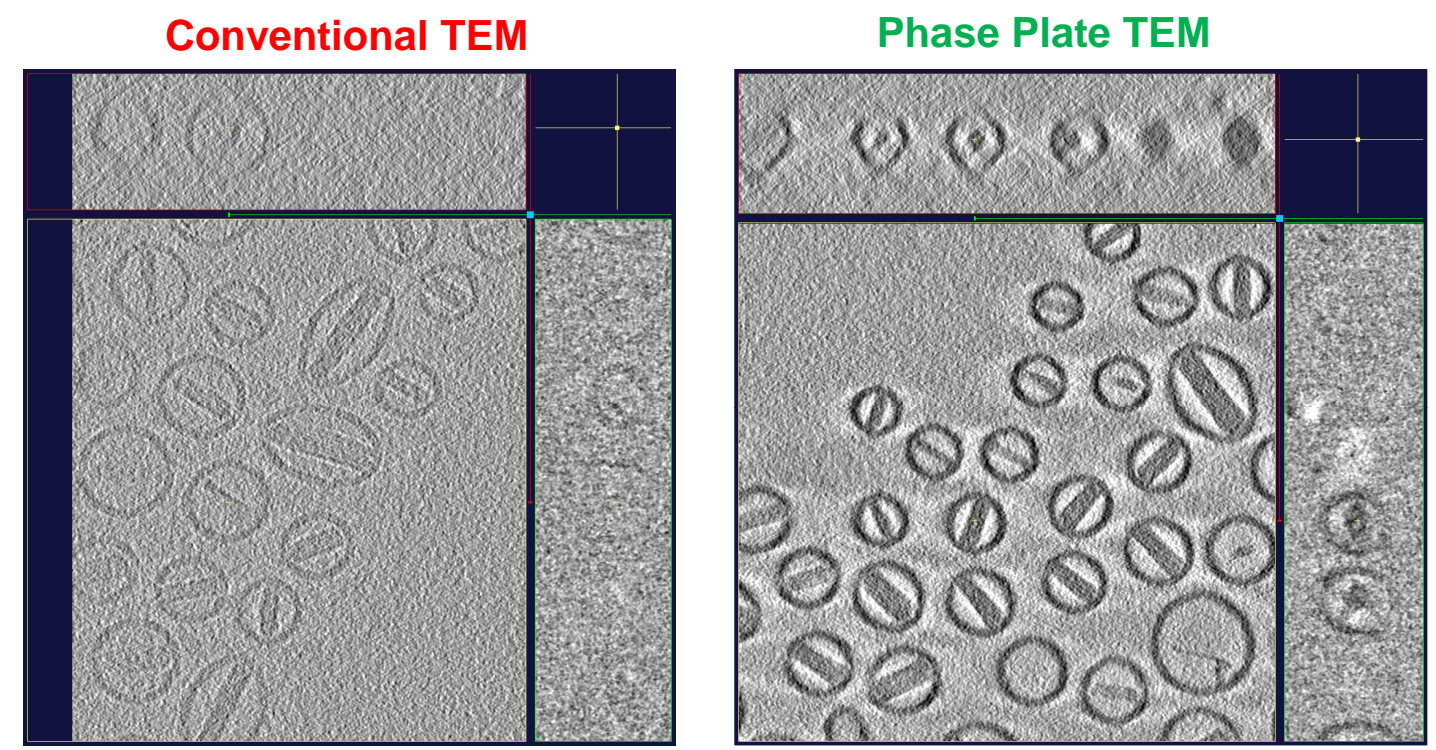

Figure 1. Tecnai F20 results from cryo electron tomography on doxorubicin using conventional TEM at $4 \mu \mathrm{m}$ defocus (left) and phase plate TEM at $0.5 \mu \mathrm{m}$ defocus (right). Experimental conditions: total dose of $85 \mathrm{e}^{-} / \AA^{2}$, tilt range $+/-60^{\circ}$. 